\title{
ON THE COEFFICIENTS OF ANALYTIC FUNCTIONS OF FAST GROWTH REPRESENTED BY DIRICHLET SERIES
}

\author{
SUNITA RANI AND G.S. SRIVASTAVA
}

1. Let $f(s)=\Sigma_{n=1}^{\infty} a_{n} e^{s \lambda_{n}}$, where $s=\sigma+i$ ( $\sigma$ and $t$ real variables), $0 \leq \lambda_{1}<$ $\lambda_{2}<\cdots, \lambda_{n} \rightarrow \infty$ as $n \rightarrow \infty$ and $\left\{a_{n}\right\}$ is a sequence of nonzero complex numbers, be a Dirichlet Series. It is well known that if

$$
\lim _{n \rightarrow \infty} \sup \frac{n}{\lambda_{n}}=D<\infty,
$$

then $f(s)$ represents an analytic function in a half plane $R e s<\alpha$, where

$$
\lim _{n \rightarrow \infty} \sup \frac{\log \left|a_{n}\right|^{-1}}{\lambda_{n}}=\alpha,-\infty<\alpha \leq \infty \text {. }
$$

We denote by $D_{\alpha}$ the class of all functions $f(s)$, analytic in the half plane $\sigma<\alpha$, $-\infty<\alpha<\infty$. Then for $f \in D_{\alpha}$, we set

$$
\begin{aligned}
M(\sigma) & =l \cdot u \cdot b_{-\infty<t<\infty}|f(\sigma+i t)|, \quad m(\sigma)=\max _{n \geq 1}\left\{\left|a_{n}\right| e^{\sigma \lambda_{n}}\right\} \\
\text { and } N(\sigma) & =\max \left\{n: m(\sigma)=\left|a_{n}\right| e^{\sigma \lambda_{n}}\right\} .
\end{aligned}
$$

Then $M(\sigma), m(\sigma)$ and $N(\sigma)$ are called respectively the maximum modulus, maximun term and the rank of the maximum term of $f(s)$.

Nandan [1] defined the order $\rho$ of $f \in D_{\alpha}$ as

$$
\lim _{\sigma \rightarrow \alpha} \sup \frac{\log \log M(\sigma)}{\log \left[\left(1-e^{\sigma-\alpha}\right)^{-1}\right]}=\rho, \quad 0 \leq \rho \leq \infty .
$$

If $\rho=\infty$, the analytic function $f$ is said to be of fast growth. For such functions, Nautiyal [2] introduced the concept of $(\beta, \delta)$ order and lower $(\beta, \delta)$ order.

Let $L^{0}$ be the class of all functions $h$ satisfying the following conditions: (i) $h(x)$ is defined on $[a, \infty)$, is positive, continuous, strictly increasing and $h(x) \rightarrow \infty$
as $x \rightarrow \infty$,

(ii) $\lim _{x \rightarrow \infty} \frac{h[x(1+\eta(x)]}{h(x)}=1$ for every function $\eta(x) \rightarrow 0$ as $x \rightarrow \infty$.

Received October 5, 1989. 
Let $\Delta$ denote the class of all functions $h$ satisfying conditions (i) and

(iii) $\lim _{x \rightarrow \infty} \frac{h(c x)}{h(x)}=1$ for every $c, 0<c<\infty$.

Evidently, the class of function $\triangle$ is a proper subset of $L^{0}$.

For a function $f \in D_{\alpha}$, set

$$
\lim _{\sigma \rightarrow \alpha} \sup _{\inf } \frac{\beta(\log M(\sigma))}{\delta(1 / 1-\exp (\sigma-\alpha))}=\frac{\rho(\beta, \delta, f)}{(\beta, \delta, f)},
$$

where $\beta \in \Delta$ and $\delta \in L^{0}$. As mentioned above, $\rho(\beta, \delta, f)$ and $\lambda(\beta, \delta, f)$ are called $(\beta, \delta)$ order and $(\beta, \delta)$ lower order of $f$ respectively. Nautiyal obtained the coefficient characterizations of $\rho(\beta, \delta)$ and $\lambda(\beta, \delta)$. We thus have

Theorem A. [2, Theorem 1]. Let $f(s) \in D_{\alpha}$ with $(\beta, \delta)$ order $\rho(\beta, \delta, f)$. Assume that

$$
\beta(x / G(x, c)) \simeq \beta(x) \text { as } x \rightarrow \infty, 0<c<\infty,
$$

where $G(x, c)=\delta^{-1}(c \beta(x))$. Then

$$
\rho(\beta, \delta, f)=\lim _{n \rightarrow \infty} \sup \frac{\beta\left(\lambda_{n}\right)}{\delta\left[\lambda_{n} / \log ^{+}\left(\left|a_{n}\right| \exp \left(\alpha \lambda_{n}\right)\right)\right]}
$$

where $\log ^{+} x=\max (0, \log x)$.

Theorem $\mathbb{B}$. [2, Lemma 6]. Let $f(s) \in D_{\alpha}$ with $(\beta, \delta)$ - order $\rho(\beta, \delta, f)>0$ and lower $(\beta, \delta)$ order $\lambda(\beta, \delta, f)$. Assume that (1.5) is satisfied and

\section{Then}

$$
\begin{aligned}
& \lim _{n \rightarrow \infty} \inf \left(\lambda_{n}-\lambda_{n-1}\right)=\theta>0, \\
& \Psi(n)=\frac{\log \left|a_{n} / a_{n+1}\right|}{\lambda_{n+1}-\lambda_{n}} \text { is a non-decreasing function of } n \text { for all } n>n_{0}, \\
& \lim _{\sigma \rightarrow \infty} \inf \frac{\beta\left(\lambda_{N(\sigma)}\right)}{\delta\left[1 /\left(1-e^{\sigma-\alpha}\right)\right]}=\lambda(\beta, \delta, f) .
\end{aligned}
$$

$$
\lim _{n \rightarrow \infty} \inf \frac{\beta\left(\lambda_{n-1}\right)}{\delta\left[\lambda_{n} / \log ^{+}\left(\left|a_{n}\right| \exp \left(\alpha \lambda_{n}\right)\right)\right]}=\lambda(\beta, \delta, f) .
$$

It is evident that for two analytic functions having same $(\beta, \delta)$ order, we need further classification to compare their growth. The authors introduced in [3] the concept of $(\beta, \delta, Y, \rho)$-type and $(\beta, \delta, Y, \rho)$ lower type for analytic functions $f(s) \in D_{\alpha}$. Hence if $0<\rho<\infty$, then we define

$$
\lim _{\sigma \rightarrow \alpha} \sup _{\inf } \frac{\beta(\log M(\sigma))}{\delta\left[\left\{\gamma\left(\frac{1}{1-e^{\sigma-\alpha}}\right)\right\}^{\rho}\right]}=\frac{T(\beta, \sigma, \gamma, \rho, f)}{t(\beta, \sigma, \gamma, \rho, f)},
$$


where $\beta, \gamma \in \triangle$ and $\delta \in L^{0}$. For simplicity, we shall denote $T(\beta, \delta, \gamma, \rho, f)$ and $t(\beta, \delta, \gamma, \rho, f)$ by $T$ and $t$ respectively. We obtained in [3] the coefficient characterizations of $T$ and $t$. Hence we have

Theorem C. Let $f(s) \in D_{\alpha}$ be of type $T$ and assume that

$$
\beta\left(\frac{x}{G(x, c, \rho)}\right) \sim \beta(x) \text { as } x \rightarrow \infty, 0<c<\infty,
$$

where $G(x, c, \rho)=\gamma^{-1}\left[\left\{\delta^{-1}\left(\frac{\beta(x)}{c}\right)\right\}^{1 / \rho}\right]$.

$$
\lim _{n \rightarrow \infty} \sup \frac{\beta\left(\lambda_{n}\right)}{\delta\left[\left\{\gamma\left(\frac{\lambda_{n}}{\log ^{+}\left|a_{n}\right|+\alpha \lambda_{n}}\right)\right\}^{\rho}\right]}=T
$$

Theorem $\mathbb{D}$. If $f(s) \in D_{\alpha}$ and $t$ is defined by (1.11) then

$$
t \geq \lim _{k \rightarrow \infty} \inf \frac{\beta\left(\lambda_{n_{k-1}}\right)}{\delta\left[\left\{\gamma\left(\frac{\lambda_{n_{k}}}{\log ^{+}\left|a_{n_{k}}\right|+\alpha \lambda_{n_{k}}}\right)\right\}^{\rho}\right]}
$$

where $\left\{n_{k}\right\}$ is any increasing sequence of positive integers, $n_{k} \rightarrow \infty$ as $k \rightarrow \infty$.

In the present paper, we shall obtain the coefficient characterizations of $\rho(\beta, \delta, f)$, $\lambda(\beta, \delta, f)$, as defined by (1.4) and $T, t$ as defined by (1.11), in terms of the ratio of the consecutive coefficients i.e. $\left|a_{n} / a_{n+1}\right|$.

2. We now prove

Theorem 1. Let $f(s) \in D_{\alpha}$ be of $(\beta, \delta)$ order $\rho(\beta, \delta, f)=\rho$. Suppose that conditions (1.5) and (1.8) are satisfied. Further, let

$$
\frac{d \log G(t, c)}{d \log t} \Rightarrow O(1) \text { as } t \rightarrow \infty \text { for any constant } c, 0<c<\infty \text {. }
$$

Then

$$
\lim _{n \rightarrow \infty} \sup \frac{\beta\left(\lambda_{n}\right)}{\delta\left[\frac{\lambda_{n}-\lambda_{n-1}}{\log ^{+}\left|a_{n} / a_{n-1}\right|+\alpha\left(\lambda_{n}-\lambda_{n-1}\right)}\right]}=\rho .
$$

Proof. Let us denote the right hand side of (2.2) by $A$ and let assume that $A<\infty$. Then for $\varepsilon>0$, there exists integer $n_{0}$ such that

$$
\beta\left(\lambda_{n}\right)<(A+\varepsilon) \delta\left[\frac{\lambda_{n}-\lambda_{n-1}}{\log ^{+}\left|a_{n} / a_{n-1}\right|+\alpha\left(\lambda_{n}-\lambda_{n-1}\right)}\right], n>n_{0}
$$


or, for all $n>n_{0}$,

$$
\log \left|a_{n} / a_{n-1}\right|<\frac{\lambda_{n}-\lambda_{n-1}}{G\left(\lambda_{n}, 1 /(A+\varepsilon)\right)}-\alpha\left(\lambda_{n}-\lambda_{n-1}\right)
$$

where $G\left(\lambda_{n}, 1 /(A+\varepsilon)\right)=\delta^{-1}\left[\beta\left(\lambda_{n}\right) /(A+\varepsilon)\right]$. Writing the above inequality for $n=$ $n_{0}+1, n_{0}+2, \ldots, k$ and adding all the inequalities thus obtained, we get

$$
\sum_{n=n_{0}+1}^{k} \log \left|a_{n} / a_{n-1}\right|<\sum_{n=n_{0}+1}^{k} \frac{\lambda_{n}-\lambda_{n-1}}{G\left(\lambda_{n}, 1 /(A+\varepsilon)\right)}-\alpha \sum_{n=n_{0}+1}^{k}\left(\lambda_{n}-\lambda_{n-1}\right)
$$

or,

$$
\log \left|a_{k}\right|+\alpha \lambda_{k}<O(1)+\sum_{n=n_{0}+1}^{k} \frac{\left(\lambda_{n}-\lambda_{n-1}\right)}{G\left(\lambda_{n}, 1 /(A+\varepsilon)\right)} .
$$

To estimate the expression on the right hand side of above inequality, we define a step function $n(t)=\lambda_{n}, \lambda_{n}<t \leq \lambda_{n+1}$ and let $F(t)=1 / G(t, 1 /(A+\varepsilon))$. Now rearranging the summation on right hand side, we have

$$
\begin{gathered}
\sum_{n=n_{0}+1}^{k} \frac{\lambda_{n}-\lambda_{n-1}}{G\left(\lambda_{n}, 1 /(A+\varepsilon)\right)} \\
=\lambda_{k} F\left(\lambda_{k}\right)-\sum_{n=n_{0}+1}^{k-1} \lambda_{n}\left\{F\left(\lambda_{n+1}\right)-F\left(\lambda_{n}\right)\right\}-\lambda_{n_{0}-1} F\left(\lambda_{n_{0}}\right) \\
=\lambda_{k} F\left(\lambda_{k}\right)-\int_{\lambda_{n_{0}}}^{\lambda_{k-1}} n(t) d F(t)-O(1) .
\end{gathered}
$$

Now

$$
\begin{aligned}
-\int_{\lambda_{n_{0}}}^{\lambda_{k-1}} n(t) d F(t) & =\int_{\lambda_{n_{0}}}^{\lambda_{k-1}} \frac{n(t)}{G^{2}(t)} G^{\prime}(t) d t \\
& =\int_{\lambda_{n_{0}}}^{\lambda_{k-1}} \frac{n(t)}{t G(t)} \cdot \frac{t G^{\prime}(t)}{G(t)} d t
\end{aligned}
$$

where $G(t)=G(t, 1 /(A+\varepsilon))$ and $G^{\prime}(t)=\frac{d G(t)}{d t}$. Now by definition, $n(t) / t<1$ and $F(t)=1 / G(t)$ is a decreasing function. Hence

$$
\begin{aligned}
-\int_{\lambda_{n_{0}}}^{\lambda_{k-1}} n(t) d F(t) & <\int_{\lambda_{n_{0}}}^{\lambda_{k-1}} \frac{t G^{\prime}(t)}{G(t)} \frac{d t}{G(t)} \\
& =\left[\int_{\lambda_{n_{0}}}^{\sqrt{\lambda_{k-1}}}+\int_{\sqrt{\lambda_{k-1}}}^{\lambda_{k-1}} \frac{t G^{\prime}(t)}{G(t)} \frac{d t}{G(t)}\right.
\end{aligned}
$$


According to assumption (2.1), $\frac{t G^{\prime}(t)}{G(t)} \rightarrow O(1)$ as $t \rightarrow \infty$. Hence we have

$$
-\int_{\lambda_{n_{0}}}^{\lambda_{k-1}} n(t) d F(t)<O(1) \frac{\left[\sqrt{\lambda_{k-1}}-\lambda_{n_{0}}\right]}{G\left(\lambda_{n_{0}}, \frac{1}{A+\varepsilon}\right)}+\frac{O(1)\left(\lambda_{k-1}-\sqrt{\lambda_{k-1}}\right)}{G\left(\sqrt{\lambda_{k-1}}, \frac{1}{A+\varepsilon}\right)}
$$

Since $G(t) \rightarrow \infty$ as $t \rightarrow \infty$, we finally get $-\int_{\lambda_{n_{0}}}^{\lambda_{k-1}} n(t) d F(t)<o\left(\lambda_{k-1}\right)$. Hence we have, since $\left\{\lambda_{k}\right\}$.

$$
\log ^{+}\left|a_{k}\right|+\alpha \lambda_{k}<O(1)+\lambda_{k} F\left(\lambda_{k}\right)+o\left(\lambda_{k}\right)
$$

or

$$
\frac{\log ^{+}\left|a_{k}\right|+\alpha \lambda_{k}}{\lambda_{k}}<\frac{1}{G\left(\lambda_{k}, \frac{1}{A+\varepsilon}\right)}+o(1), \quad k>n_{0}
$$

or, using the definition of $G\left(\lambda_{k}, \frac{1}{A+\varepsilon}\right)$, we have

$$
\frac{\beta\left(\lambda_{k}\right)}{\delta\left[\frac{\lambda_{k}}{\log ^{+}\left|a_{k}\right|+\alpha \lambda_{k}}\right]}<A+\varepsilon, \quad k>n_{0} .
$$

Now proceeding to limits as $k \rightarrow \infty$, we get in view of (1.6),

$$
\rho(\beta, \delta, f) \leq A
$$

The above inequality obviously holds if $A=\infty$.

To obtain the reverse inequality, we use the condition (1.8). Then, for any $n>n_{0}$,

$$
\begin{aligned}
\log \left|a_{n_{0}} / a_{n}\right| & =\log \left|\frac{a_{n_{0}}}{a_{n_{0}+1}} \cdots \frac{a_{n-1}}{a_{n}}\right| \\
& =\sum_{k=n_{0}}^{n-1}\left(\lambda_{k+1}-\lambda_{k}\right) \Psi(k) \\
& \leq \Psi(n-1) \cdot\left(\lambda_{n}-\lambda_{n_{0}}\right)
\end{aligned}
$$

since $\Psi(k)$ is a non decreasing function of $k$. Hence we have

$$
\log ^{+}\left|a_{n}\right| \geq O(1)+\frac{\lambda_{n}-\lambda_{n_{0}}}{\lambda_{n}-\lambda_{n-1}} \log ^{+}\left|a_{n} / a_{n-1}\right|
$$

or

$$
\alpha+\frac{\log ^{+}\left|a_{n} / a_{n-1}\right|}{\lambda_{n}-\lambda_{n-1}} \leq \alpha+\frac{\log ^{+}\left|a_{n}\right|}{\lambda_{n}}-o(1)
$$


i.e.

$$
\frac{\lambda_{n}}{\alpha \lambda_{n}+\log ^{+}\left|a_{n}\right|} \leq \frac{\lambda_{n}-\lambda_{n-1}}{\alpha\left(\lambda_{n}-\lambda_{n-1}\right)+\log ^{+}\left|a_{n} / a_{n-1}\right|}
$$

Since $\delta$ is an increasing function, hence we get

$$
\lim _{n \rightarrow \infty} \sup \frac{\beta\left(\lambda_{n}\right)}{\delta\left[\frac{\left(\lambda_{n}-\lambda_{n-1}\right)}{\log ^{+}\left|a_{n} / a_{n-1}\right|+\alpha\left(\lambda_{n}-\lambda_{n-1}\right)}\right]} \leq \lim _{n \rightarrow \infty} \sup \frac{\beta\left(\lambda_{n}\right)}{\delta\left[\frac{\lambda_{n}}{\left.\log ^{+}\left|a_{n}\right|+\alpha \lambda_{n}\right]}\right.}
$$

i.e. $\quad A \leq \rho(\beta, \delta, f)$.

Combining the above inequality with (2.3), we get (2.2). This completes proof of Theorem 1.

Next we prove

Theorem 2. Let $f(s) \in D_{\alpha}$ be of lower $(\beta, \delta)$ order $\lambda(\beta, \delta, f)=\lambda$. If $f(s)$ satisfies (1.9) then

$$
\lambda=\max _{\left\{n_{m}\right\}} \lim _{m \rightarrow \infty} \inf \frac{\beta\left(n_{m-1}\right)}{\delta\left[\left(\lambda_{n_{m}}-\lambda_{n_{m-1}}\right) / \log ^{+}\left|a_{n_{m}} / a_{n_{m-1}}\right|+\alpha\left(\lambda_{n_{m}}-\lambda_{n_{m-1}}\right)\right]}
$$

where maximum on the right hand side of (2.5) is taken over all increasing sequences of natural numbers $\left\{n_{m}\right\}$ such that $n_{m} \rightarrow \infty$ as $m \rightarrow \infty$.

Proof. Let the limit inferior on the right hand side of (2.5) be denoted by $B$. Clearly $0 \leq B \leq \infty$. First let $0<B<\infty$. Then for any $\varepsilon>0$ and all integers $k>N$, we have

Or

$$
\beta\left(\lambda_{n_{k-1}}\right)>(B-\varepsilon) \delta\left[\frac{\lambda_{n_{k}}-\lambda_{n_{k-1}}}{\log ^{+}\left|a_{n_{k}} / a_{n_{k-1}}\right|+\alpha\left(\lambda_{n_{k}}-\lambda_{n_{k-1}}\right)}\right]
$$

$$
\log \left|a_{n_{k}} / a_{n_{k-1}}\right|>\frac{\lambda_{n_{k}}-\lambda_{n_{k-1}}}{G\left(\lambda_{n_{k-1}}, 1 /(B-\varepsilon)\right)}-\alpha\left(\lambda_{n_{k}}-\lambda_{n_{k-1}}\right)
$$

where $G\left(\lambda_{n_{k-1}}, 1 /(B-\varepsilon)\right)=\delta^{-1}\left[\beta\left(\lambda_{n_{k-1}}\right) /(B-\varepsilon)\right]$.

Writing above inequality for $k=N, N+1, \cdots, m$ and adding all the inequalities thus obtained, we get

$$
\sum_{k=N}^{m} \log \left|a_{n_{k}} / a_{n_{k-1}}\right|>\sum_{k=N}^{m} \frac{\lambda_{n_{k}}-\lambda_{n_{k-1}}}{G\left(\lambda_{n_{k-1}}, 1 /(B-\varepsilon)\right)}-\alpha\left(\lambda_{n_{m}}-\lambda_{n_{N-1}}\right)
$$

or,

$$
\log \left|a_{n_{m}}\right|+\alpha \lambda_{n_{m}}>O(1)+\sum_{k=N}^{m} \frac{\lambda_{n_{k}}-\lambda_{n_{k-1}}}{G\left(\lambda_{n_{k-1}}, 1 /(B-\varepsilon)\right)} .
$$

Since $1 / G(t)$ is a decreasing function therefore

$$
\log ^{+}\left|a_{n_{m}}\right|+\alpha \lambda_{n_{m}}>O(1)+\frac{\lambda_{n_{m}}-\lambda_{n_{N-1}}}{G\left(\lambda_{n_{m-1}}, 1 /(B-\varepsilon)\right)}
$$


or

$$
G\left(\lambda_{n_{m-1}}, 1 /(B-\varepsilon)\right)>\frac{\lambda_{n_{m}}}{\log ^{+}\left|a_{n_{m}}\right|+\alpha \lambda_{n_{m}}}+o(1), \quad m>N .
$$

On using the definition of $G\left(\lambda_{n_{m-1}}, 1 /(B-\varepsilon)\right)$, and proceeding to limits, we have

$$
\begin{aligned}
B & \leq \lim _{m \rightarrow \infty} \inf \frac{\beta\left(\lambda_{n_{m-1}}\right)}{\delta\left[\lambda_{n_{m}} /\left(\log ^{+}\left|a_{n_{m}}\right|+\alpha \lambda_{n_{m}}\right)\right]} \\
& \leq \lambda(\beta, \delta, f) \quad \text { (from Lemma } 6,[2]) .
\end{aligned}
$$

Since $\left\{n_{m}\right\}$ was any arbitrary sequence of positive integers, we get

$$
\lambda(\beta, \delta, f) \geq \max _{\left\{n_{m}\right\}} \lim _{m \rightarrow \infty} \inf \frac{\beta\left(\lambda_{n_{m-1}}\right)}{\delta\left[\left(\lambda_{n_{m}}-\lambda_{n_{m-1}}\right) /\left\{\log ^{+}\left|a_{n_{m}} / a_{n_{m-1}}\right|+\alpha\left(\lambda_{n_{m}}-\lambda_{n_{m-1}}\right)\right\}\right]} .
$$

To prove the reverse inequality, let the range of the $\operatorname{rank} N(\sigma)$ be the sequence $\left\{n_{k}\right\}$. Also, let $\Psi(n)$ denote the jump points of $N(\sigma)$. Then

$$
\begin{aligned}
N(\sigma) & =n_{k} \text { for } \Psi\left(n_{k}\right) \leq \sigma<\Psi\left(n_{k+1}\right), \quad k=1,2, \cdots, \text { where } \\
\Psi\left(n_{k}\right) & =\frac{\log \left|a_{n_{k-1}} / a_{n_{k}}\right|}{\lambda_{n_{k}}-\lambda_{n_{k-1}}} .
\end{aligned}
$$

Now, under the assumption (1.9) we have

$$
\begin{aligned}
\lambda(\beta, \delta, f) & =\lim _{\sigma \rightarrow \alpha} \inf \frac{\beta\left(\lambda_{N(\sigma)}\right)}{\delta\left\{\left(1-e^{\sigma-\alpha}\right)^{-1}\right\}} \\
& =\lim _{k \rightarrow \infty} \inf \frac{\beta\left(\lambda_{n_{k}}\right)}{\delta\left[\left\{1-\exp \left(\Psi\left(n_{k+1}\right)-\alpha\right)\right\}^{-1}\right]}
\end{aligned}
$$
It can be easily seen that $1-e^{\sigma-\alpha} \simeq \alpha-\sigma$ as $\sigma \rightarrow \alpha$. Hence in view of property (ii) of
$\delta$, we get

Hence

$$
\begin{aligned}
\lambda(\beta, \delta, f) & =\lim _{k \rightarrow \infty} \inf \frac{\beta\left(\lambda_{n_{k-1}}\right)}{\delta\left[\left\{\alpha-\Psi\left(n_{k}\right)\right\}^{-1}\right]} \\
& =\lim _{k \rightarrow \infty} \inf \frac{\beta\left(\lambda_{n_{k-1}}\right)}{\delta\left[\left(\lambda_{n_{k}}-\lambda_{n_{k-1}}\right) /\left\{\log ^{+}\left|a_{n_{k}} / a_{n_{k-1}}\right|+\alpha\left(\lambda_{n_{k}}-\lambda_{n_{k-1}}\right)\right\}\right]}
\end{aligned}
$$

$$
\lambda(\beta, \delta, f) \leq \max _{\left\{n_{m}\right\}} \lim _{m \rightarrow \infty} \inf \frac{\beta\left(\lambda_{n_{m-1}}\right)}{\left.\delta\left[\lambda_{n_{m}}-\lambda_{n_{m-1}}\right) /\left\{\log ^{+}\left|a_{n_{m}}\right|+\alpha\left(\lambda_{n_{m}}-\lambda_{n_{m-1}}\right)\right\}\right]} .
$$

Combining the above inequality with (2.6) we get (2.5). This completes the proof of
Theorem 2. 
3. In this section, we shall obtain coefficient characterization of the type $T$ and lower type $t$ as defined by (1.11). We prove

Theorem 3. Let $f(s) \in D_{\alpha}$ be of $(\beta, \delta, \gamma, f)$-type $T$ and lower $(\beta, \delta, \gamma, f)$ type $t$. Suppose that the condition (1.8) and (1.12) are satisfied. Then

$$
T=\lim _{n \rightarrow \infty} \sup \frac{\beta\left(\lambda_{n}\right)}{\delta\left[\left\{\gamma\left(\frac{\lambda_{n}-\lambda_{n-1}}{\log ^{+}\left|a_{n} / a_{n-1}\right|+\alpha\left(\lambda_{n}-\lambda_{n-1}\right)}\right)\right\}^{\rho}\right]} .
$$

Proof. Let the expression on the right hand side of (3.1) be denoted by $Q$. Clearly $0 \leq Q \leq \infty$. First let $0<Q<\infty$. Then for $\varepsilon>0$ we have for all sufficiently large $n>N(\varepsilon)$,

or, for all $n>N$,

$$
\beta\left(\lambda_{n}\right)<(Q+\varepsilon) \delta\left[\left\{\gamma\left(\frac{\lambda_{n}-\lambda_{n-1}}{\log ^{+}\left|a_{n} / a_{n-1}\right|+\alpha\left(\lambda_{n}-\lambda_{n-1}\right)}\right)\right\}^{\rho}\right]
$$

$$
\log \left|a_{n} / a_{n-1}\right|<\frac{\lambda_{n}-\lambda_{n-1}}{G\left(\lambda_{n}, 1 /(Q+\varepsilon), \rho\right)}-\alpha\left(\lambda_{n}-\lambda_{n-1}\right),
$$

where $G\left(\lambda_{n}, 1 / Q+\varepsilon, \rho\right)=\gamma^{-1}\left[\left\{\delta^{-1}\left(\frac{\beta\left(\lambda_{n}\right)}{Q+\varepsilon}\right)\right\}^{1 / \rho}\right]$.

Writing the above inequality for $n=N+1, N+2, \cdots, k$ and adding we get

$$
\sum_{n=N+1}^{k} \log \left|a_{n} / a_{n-1}\right|<\sum_{n=N+1}^{k} \frac{\left(\lambda_{n}-\lambda_{n-1}\right)}{G\left(\lambda_{n}, 1 / Q+\varepsilon, \rho\right)}-\alpha\left(\lambda_{k}-\lambda_{N}\right)
$$

or,

$$
\log \left|a_{k}\right|+\alpha \lambda_{k}<O(1)+\sum_{n=N+1}^{k} \frac{\lambda_{n}-\lambda_{n-1}}{G\left(\lambda_{n}, \frac{1}{Q+\varepsilon}, \rho\right)} .
$$

Let us write $F(t)=\frac{1}{G\left(\lambda_{i}, 1 / Q+\varepsilon, \rho\right)}$ and $n(t)=\lambda_{n}$ for $\lambda_{n}<t \leq \lambda_{n+1}$. Then we have $\frac{n(t)}{t}<1$. We consider

$$
\begin{aligned}
\sum_{n=N+1}^{k} \frac{\lambda_{n}-\lambda_{n-1}}{G\left(\lambda_{n}, 1 / Q+\varepsilon, \rho\right)} & =\lambda_{k} F\left(\lambda_{k}\right)-\lambda_{N} F\left(\lambda_{N+1}\right)-\sum_{n=N+1}^{k-1} \lambda_{n}\left[F\left(\lambda_{n+1}\right)-F\left(\lambda_{n}\right)\right] \\
& =\lambda_{k} F\left(\lambda_{k}\right)-\lambda_{N} F\left(\lambda_{N+1}\right)-\int_{\lambda_{N+1}}^{\lambda_{k-1}} n(t) d F(t) .
\end{aligned}
$$

As in the proof of Theorem 1, we can easily show that

$$
-\int_{\lambda_{N+1}}^{\lambda_{k-1}} n(t) d F(t)=o\left(\lambda_{k-1}\right) \text {. }
$$


Hence we have for all large $k$,

$$
\log ^{+}\left|a_{k}\right|+\alpha \lambda_{k}<\frac{\lambda_{k}}{G\left(\lambda_{k}, 1 / Q+\varepsilon, \rho\right)}(1+o(1)), \quad k>N
$$

or, using the definition of $G\left(\lambda_{k}, 1 / Q+\varepsilon, \rho\right)$, we have

$$
\frac{\beta\left(\lambda_{k}\right)}{\delta\left[\left\{\gamma\left(\lambda_{k} / \log ^{+}\left|a_{k}\right|+\alpha \lambda_{k}\right)\right\}^{\rho}\right]}<Q+\varepsilon, \quad k>N .
$$

Now proceeding to limits as $k \rightarrow \infty$, we get in. view of (1.13),

$$
T=T(\beta, \delta, \gamma, \rho, f) \leq Q
$$

The above inequality obviously holds if $Q=\infty$. To prove the reverse inequality, we have from (2.4),

or,

$$
\alpha+\mid \frac{\log ^{+}\left|a_{n} / a_{n-1}\right|}{\lambda_{n}-\lambda_{n-1}} \leq \alpha+\frac{\log ^{+}\left|a_{n}\right|}{\lambda_{n}}-o(1)
$$

or,

$$
\frac{\alpha\left(\lambda_{n}-\lambda_{n-1}\right)+\log ^{+}\left|a_{n}\right| a_{n-1} \mid}{\lambda_{n}-\lambda_{n-1}} \leq \frac{\log ^{+}\left|a_{n}\right|+\alpha \lambda_{n}}{\lambda_{n}}
$$

$$
\delta\left[\left\{\gamma\left(\frac{\lambda_{n}-\lambda_{n-1}}{\alpha\left(\lambda_{n}-\lambda_{n-1}\right)+\log ^{+}\left|a_{n} / a_{n-1}\right|}\right)\right\}^{\rho}\right] \geq \delta\left[\left\{\gamma\left(\frac{\lambda_{n}}{\log ^{+}\left|a_{n}\right|+\alpha \lambda_{n}}\right)\right\}^{\rho}\right] .
$$

Therefore

$$
\frac{\beta\left(\lambda_{n}\right)}{\delta\left[\left\{\gamma\left(\frac{\lambda_{n}}{\log ^{+}\left|a_{n}\right|+\alpha \lambda_{n}}\right)\right\}^{\rho}\right]} \geq \frac{\beta\left(\lambda_{n}\right)}{\delta\left[\left\{\gamma\left(\frac{\left(\lambda_{n}-\lambda_{n-1}\right)}{\log ^{+}\left|a_{n} / a_{n-1}\right|+\alpha\left(\lambda_{n}-\lambda_{n-1}\right.}\right)\right\}^{\rho}\right]}
$$

which gives, on taking limits

$$
T \geq Q
$$

Again this inequality holds if $Q=0$. Now combining (3.2) with (3.3), we get (3.1). This completes proof of Theorem 3 .

Lastly we prove

Theorem 4. Let $f(s) \in D_{\alpha}$ of be of lower $(\beta, \delta, \gamma, \rho)$ type $t(\beta, \delta, \gamma, \rho, f)$ and satisfy condition (1.12). Then for any increasing sequence $\left\{n_{k}\right\}$ of positive integers, we have

$$
t \geq \lim _{k \rightarrow \infty} \inf \frac{\beta\left(\lambda_{n_{k-1}}\right)}{\delta\left[\left\{\gamma\left(\frac{\lambda_{n_{k}}-\lambda_{n_{k-1}}}{\log ^{+}\left|a_{n_{k}} / a_{n_{k-1}}\right|+\alpha\left(\lambda_{n_{k}}-\lambda_{n_{k-1}}\right)}\right)\right\}^{\rho}\right]}
$$

Proof. Let us denote the right hand side of (3.4) by $q$. Clearly $0 \leq q \leq \infty$. First let $0<q$. Then for all $k>N, \varepsilon>0$, we have 
where

$$
G\left(\lambda_{n_{k-1}}, \frac{1}{q-\varepsilon}, \rho\right)>\frac{\lambda_{n_{k}}-\lambda_{n_{k-1}}}{\log ^{+}\left|a_{n_{k}} / a_{n_{k-1}}\right|+\alpha\left(\lambda_{n_{k}}-\lambda_{n_{k-1}}\right)}
$$

Or,

$$
G\left(\lambda_{n_{k-1}}, \frac{1}{q-\varepsilon}, \rho\right)=\gamma^{-1}\left[\left\{\delta^{-1}\left(\frac{\beta\left(\lambda_{n_{k-1}}\right)}{q-\varepsilon}\right)\right\}^{1 / \rho}\right]
$$

$$
\log ^{+}\left|a_{n_{k}} / a_{n_{k-1}}\right|>\frac{\lambda_{n_{k}}-\lambda_{n_{k-1}}}{G\left(\lambda_{n_{k-1}}, 1 / q-\varepsilon, \rho\right)}-\alpha\left(\lambda_{n_{k}}-\lambda_{n_{k-1}}\right) .
$$
Writing the above inequality for $m=N, N+1, \cdots, k$ and adding all these inequalities,
we get

$$
\log ^{+}\left|a_{n_{k}}\right|+\alpha \lambda_{n_{k}}>\sum_{m=N}^{k} \frac{\lambda_{n_{m}}-\lambda_{n_{m-1}}}{G\left(\lambda_{n_{m-1}}, 1 / q-\varepsilon, \rho\right)}+O(1) .
$$

As in the proof of Theorem 2. we have

$$
\log ^{+}\left|a_{n_{k}}\right|+\alpha \lambda_{n_{k}}>\frac{\lambda_{n_{k}}-\lambda_{n_{N-1}}}{G\left(\lambda_{n_{k-1}}, 1 / q-\varepsilon, \rho\right)}+O(1)
$$

or,

$$
G\left(\lambda_{n_{k-1}}, 1 / q-\varepsilon, \rho\right)>\frac{\lambda_{n_{k}}}{\log ^{+}\left|a_{n_{k}}\right|+\alpha \lambda_{n_{k}}}+o(1) .
$$

Hence proceding to limits, we get on using (1.14),

$$
q \leq \lim _{k \rightarrow \infty} \inf \frac{\beta\left(\lambda_{n_{k-1}}\right)}{\delta\left[\left\{\gamma\left(\lambda_{n_{k}} / \log ^{\dagger}\left|a_{n_{k}}\right|+\alpha \lambda_{n_{k}}\right)\right\}^{\rho}\right]}
$$

or,

$$
t \geq \lim _{k \rightarrow \infty} \inf \frac{\beta\left(\lambda_{n_{k-1}}\right)}{\delta\left[\left\{\gamma\left(\lambda_{n_{k}}-\lambda_{n_{k-1}} /\left(\log ^{+}\left|a_{n_{k}} / a_{n_{k-1}}\right|+\alpha\left(\lambda_{n_{k}}-\lambda_{n_{k-1}}\right)\right)\right\}^{\rho}\right]\right.} .
$$

This proves Theorem 4.

\section{References}

[1] K. Nandan, "On the maximum term and.maximum modulus of analytic functions represented by Dirichlet series," Ann. Polon Math. 28 (1973), 213-222. [2] A. Nautiyal, "On the coefficients of analytic Dirichlet series of fast growth," Indian J. pure appl.
Math. 15(10) (1984), 1102-14. [3] G.S. Srivastava and Sunita Rani, Coefficients of analytic functions of fast growth represented by
Dirichlet series, Communicated for publication. 